\title{
Palaeoenvironmental controls on the distribution of Cretaceous herbivorous dinosaurs
}

\author{
Richard J. Butler • Paul M. Barrett
}

Published online: 6 August 2008

(C) Springer-Verlag 2008

\section{Erratum to : Naturwissenschaften}

DOI 10.1007/s00114-008-0417-5

Table 1 of the paper "Palaeoenvironmental controls on the distribution of Cretaceous herbivorous dinosaurs" by Richard J. Butler and Paul M. Barrett contains an error. The significant correlation between the dinosaur clade Hadrosauridae and the 'Terrestrial' environmental category is indicated as being positive in nature; this correlation is actually a negative correlation as indicated correctly in the text (page 3). A corrected version of Table 1 follows.

The online version of the original article can be found at http://dx.doi. org/10.1007/s00114-008-0417-5.

R. J. Butler $(\bowtie) \cdot$ P. M. Barrett

Department of Palaeontology, The Natural History Museum,

Cromwell Road,

London SW7 5BD, UK

e-mail: r.butler@nhm.ac.uk 
Table 1 Results of the Chi-squared tests of association between depositional environments and dinosaur groups

\begin{tabular}{|c|c|c|c|c|c|c|c|c|c|}
\hline & \multicolumn{3}{|c|}{ "Marine" (136) } & \multicolumn{3}{|c|}{ “Coastal” (225) } & \multicolumn{3}{|c|}{ “Terrestrial” (1482) } \\
\hline & $x^{2}$ & $+/-$ & $\mathrm{p}$ & $x^{2}$ & $+/-$ & $\mathrm{p}$ & $x^{2}$ & $+/-$ & $\mathrm{p}$ \\
\hline Ankylosauria (240) & $7.46_{(28)}$ & + & $0.006^{* *}$ & $0.43_{(33)}$ & N/A & 0.61 & $5.95_{(179)}$ & - & $0.015^{*}$ \\
\hline Nodosauridae (90) & $4.91_{(12)}$ & + & $0.027 *$ & $2.74_{(16)}$ & N/A & 0.098 & $7.98_{(62)}$ & - & $0.005^{* *}$ \\
\hline Ankylosauridae (79) & $1.55_{(3)}$ & N/A & 0.213 & $2.66_{(5)}$ & N/A & 0.103 & $4.69_{(71)}$ & + & $0.03 *$ \\
\hline Ornithopoda (ichnology) (72) & $\mathrm{N} / \mathrm{A}$ & N/A & N/A & $0.272_{(46)}$ & N/A & 0.602 & $0.272_{(46)}$ & N/A & 0.602 \\
\hline Non-hadrosaurid Ornithopoda (321) & $0.03_{(23)}$ & N/A & 0.872 & $11.17_{(57)}$ & + & $0.008 * *$ & $7.02_{(241)}$ & - & $0.008 * *$ \\
\hline Hadrosauridae (391) & $43.17_{(59)}$ & + & $<0.0001 * *$ & $0.84_{(53)}$ & N/A & 0.359 & $25.85_{(279)}$ & - & $<0.0001^{* *}$ \\
\hline Hadrosaurinae (111) & $1.43_{(5)}$ & N/A & 0.232 & $2.76_{(8)}$ & N/A & 0.097 & $4.65_{(98)}$ & + & $0.031^{*}$ \\
\hline Lambeosaurinae (59) & $0.61_{(3)}$ & N/A & 0.436 & $1.03_{(5)}$ & N/A & 0.311 & $1.41_{(51)}$ & $\mathrm{N} / \mathrm{A}$ & 0.236 \\
\hline Pachycephalosauria (69) & $5.71_{(0)}$ & - & $0.017^{*}$ & $1.65_{(5)}$ & N/A & 0.199 & $6.93_{(64)}$ & + & $0.008 * *$ \\
\hline Ceratopsia (353) & $32.17_{(1)}$ & - & $<0.0001 * *$ & $1.65_{(36)}$ & N/A & 0.2 & $22.99_{(316)}$ & + & $<0.0001^{* *}$ \\
\hline Chasmosaurinae (113) & $7.43_{(1)}$ & - & $0.006^{* *}$ & $0.004_{(14)}$ & N/A & 0.952 & $3.05_{(98)}$ & N/A & 0.081 \\
\hline Sauropoda - ichnology only (27) & N/A & N/A & N/A & $0.8_{(9)}$ & N/A & 0.8 & $0.8_{(18)}$ & N/A & 0.8 \\
\hline Sauropoda - total-evidence (354) & $1.34_{(21)}$ & N/A & 0.247 & $4.87_{(31)}$ & - & $0.027 *$ & $6.68_{(302)}$ & + & $0.0097 * *$ \\
\hline Macronaria (190) & $3.11_{(8)}$ & $\mathrm{N} / \mathrm{A}$ & 0.08 & $14.36_{(7)}$ & - & $0.0002 * *$ & $18.38_{(175)}$ & + & $<0.0001 * *$ \\
\hline Herbivorous theropods (94) & $4.00_{(2)}$ & - & $0.046^{*}$ & $2.1_{(7)}$ & $\mathrm{N} / \mathrm{A}$ & 0.148 & $6.35_{(85)}$ & + & $0.012 *$ \\
\hline
\end{tabular}

